\title{
Inhaltsübersicht der El-Amarna-Tafeln
}

in der Bearbeitung von J. A. Knudtzon.

I. Briefe:

Abdi-Aširte (bezw. Ašratu) an den König. Nr. 60. 6r.

", , an Pahanate. Nr. 62.

Abdi-Ašta(r)ti an den König. Nr. 63. 64; vgl. auch Nr. 66?

Abdihiba von Jerusalem an den König. 6 oder 7 Briefe: Nr. 285-29o; vielleicht auch $\mathrm{Nr} .29 \mathrm{I}$.

Abdimilki von Šashimi an den König. Nr. 203.

Abdi-na _. - a an den König. Nr. 229.

Abdi-tirši von Hazur an den König. Nr. 228.

Abimilki von Tyrus an den König. Io Briefe: Nr. 146-I55.

Addu- ..... an den König. Nr. 249. 250.

Addu-dâni an den König. Nr. 292-295?

Addu-nirari an den König von Ägypten. Nr. 5 r.

Adra-Aštarti (= Abdi-A.?) an den König. Nr. 65 .

Ägyptens König an den Fürsten von Ammia(?). Nr. 99.

", (bezw. Hof) an den Fürsten von Amurra. Nr. 162; wohl auch 163 .

", (bezw. Hof) an Etakkama? Nr. Igo.

$A[k \ldots+]$ an den König. Nr. 217.

Akizzi von Kațna an Amenophis IIl. Nr. 52-55.

Alasia-König an den König von Ägypten. 7 Briefe: Nr. $33-39$.

Alasia-rabiș an den rabiș von Ägypten. Nr. 40.

Amajaše an den König. Nr. 202.

Amenophis III an Kadašman-Harbe. Nr. r. 5.

", an Tarhundaraba, meistens in der Arzawa-Sprache. Nr. $3 \mathbf{t}$.

Ammunira von Bêrût an den König. Nr. 141-143.

Ara[ba]ttu - von Kumidi an den König. Nr. 198.

Artamanja von Ziribašani an den König. Nr. 201.

Arzawa-Brief Nr. 2 (vgl. Amenophis III an Tarbuundaraba). Nr. 32.

Arzawija von Ruhiza an den König. Nr. 191. 192.

Ašur-uballiț an Amenophis IV. Nr. 15. 16.

Aziri an Dûdu. Nr. 158. 164.

", an Hâi. Nr. 166. 167(?).

", an den König. 7 Briefe: Nr. 156. 157. 159-16r. 165. I68.

Aziri-Sohn an einen hohen ägypt. Beamten (Hâi?). Nr. I69.

Aziri-Sohn(?) an den König. Nr. I71.

Babylonische (vgl. S. 20) Prinzessin an ihren Herrn. Nr. I2.

Baduzan[a] an den König. Nr. 239. 


\section{Inhaltsübersicht.}

Bajadi an einen Großen. Nr. 238.

", an den König. Nr. 237.

Baiawa an den König. Nr. 215. 216.

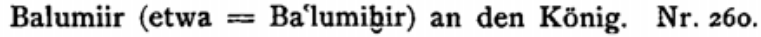

Ba'aluia und Battiilu an den König. Nr. 170.

Ba'lat- ..... an den König. Nr. 273. 274.

$B a^{c} l u$-mihir an den König. Nr. 257-259.

Bieri von Hašabu an den König. Nr. 174.

Biridija von Megiddo an den König. 5 Briefe: Nr. 242-246.

Biridija oder Jašdata an den König. Nr. 247.

Burraburiaš an Amenophis III(?). Nr. 6.

", an Amenophis IV. 5 Briefe: Nr. 7-rr.

Cölesyrische (vgl. S. 57 oben) verstümmelte Briefe an den König. Nr. 176.

179-181. 188; etwa auch Nr. 172 (vgl. unten unter 2), 173 u. 199.

Dagantakala an den König. Nr. 317. 318.

Dašru an den König. Nr. 26r. 262.

„Diener" an den König. . Nr. 200.

Dijate an den König. Nr. 193.

$E$ bi od. [P]âbi an einen Großen. Nr. 333.

$\operatorname{En}[g]$ û[t]a an den König. Nr. 223.

Etakkama an den König. Nr. 189.

Gubbu-Fürst an den König. Nr. 205.

Gubla (Stadt) siehe unter Ilirabih.

Gubla-Mann an den König. Nr. 94.

", an einen hohen ägypt. Beamten oder den König. Nr. Ior.

Hatti(?)-König an den König von Ägypten^ Nr. 42.

Hazur-König (vgl. Abdi-tirši) an den König. Nr. 227.

Hibija an einen Großen. Nr. 178.

Hiziri an den König. Nr. 336. 337.

Iabniilu von Lakiš an den König. Nr. 328.

Ia[h]tiri an den König. Nr. 296.

Iabzibada an den König. Nr. 275. 276.

Iama an den König. Nr. 230.

Jamiuta von Guddašuna an den König. Nr. 177.

Iapah-Addi an Ianbamu. Nr. 98.

Iapabi von Gazri an den König. Nr. 297-299; wahrscheinlich auch 300.

Iappa[ $b$-Addi] an Sumu-badi. Nr. 97.

$\mathrm{Ja}[\mathrm{šd}] a t a$ an den König. Nr. 248; siehe auch Biridija.

Ildâja von Hazi an den König. Nr. 175 .

Ilirabih und die Stadt Gubla an den König. Nr. 139. 140.

Ipte _ _ - an den König. Nr. 207.

Irḳata (Stadt) an den König. Nr. 100.

Kadašman-Harbe an Amenophis III. Nr. 2-4.

Labaja an den König. Nr. 252-254.

Majarzana von Hazi an den König. Nr. 185. 186.

Milkili an den König. 5 Briefe: Nr. 267-27I.

$[M]$ istu _ _ an den König. Nr. 45. 


\section{Inhaltsübersicht.}

Mittelpalästinensische(?) unvvollständige Briefe. Nr. 251 an einen hohen ägypt. Beamten; Nr. 263 wohl an den König.

Mut-Baclu an Ianbamu. Nr. 256.

", an den König. Nr. 255.

Namiawaza an den König. Nr. 194-r97.

Naziba-Fürst an den König. Nr. 206.

Nordpalästinerisische(?; vgl. S. 58) verstümmelte Briefe an den König. Nr. 214. 218. 219. 231; etwa auch Nr. 208. 236. 240.

Nordsyrischer(?) König an den König von Ägypten. Nr. 43. ", an die Könige Kanaans. Nr. 30.

Nordsyrische (so m. E.) verstümmelte Briefe: a) Nr. 46 und 47 , beide an den König, mit dem Brief von $[M]$ istu _... (Nr. 45) eng verwandt; b) Nr. 56 und 57, sich eng an die Briefe Akizzis (Nr. 52-55) anschließend.

Nukurtuwa von(?) $[Z] u$ nu an den König. Nr. 220.

"Oberst" an Rib-Addi. Nr. 96.

[P]âbi siehe $E$ bi.

Phönizischer(?) verstümmelter Brief an den König. Nr. 67 .

$\mathrm{Pu}-\mathrm{Ba} l u$ von Jurșa an den König. Nr. 314. 315 .

", an den König und an Sahšibgšsiha. Nr. 316 .

Kanû-Fürst an den König. Nr. 204.

[Kat]ihutišupa an den König(?). Nr. 58.

Rib-Addi an Amanappa. 6 Briefe: Nr. 73. 77. 82. 86. 87. 93.

", an einen hohen ägypt. Beamten. Nr. 69. I13 (letzterer Brief doch vielleicht an den König).

", an einen Großen. Nr. 95.

", an Haia (?). Nr. $7 \mathbf{r}$.

$"$, an [Ianka] $m[u]$. Nr. 102.

$" "$ an den König. 53 Briefe: Nr. 68. 7o. 72. 74-76. $78-81.83-85$. 88-92. 103-112. $114-138$.

Rușmania von Şaruna an den König. Nr. 241.

Šama-Adda an den König. Nr. 49.

Šamu-Adda von Šambuna an den König. Nr. 225.

Šatija an den König. Nr. 187.

Šipti-Ba $l u$ an den König. Nr. 330. 331; wohl auch 332.

Šiptur[i _] an den König. Nr. 226.

Šubandu an den König. 6 Briefe: Nr. 301-306.

Subbiluliuma an Hûrî[ $a]$. Nr. 4r.

Šum-add $[a]$ an den König. Nr. 224.

Šum _. _ an den König. Nr. 272.

Sutarna von Mušihuna an den König. Nr. 182-184.

Suwardata an den König. 7 Briefe: Nr. 278-284.

Südpalästinensische verstümmelte Briefe an den König (so wenigstens die meisten). Nr. 277 (von Iahzibada?), Nr. 307-313 (einige von diesen etwa von Šubandu) und Nr. 327 (von Widia?).

Tagi an den König. Nr. 264. 265; auch 266?

"Tochter von _ -" an ihre Herrin $B\left[i_{\text {_ }}\right.$ - ] (vgl. S. 2 I oben). Nr. 50. 


\section{Inhaltsübersicht.}

Tunips Einwohner an den König. Nr. 59.

Tušratta an Amenophis III. 7 Briefe: Nr. 17-21. 23. 24 (dieser letzte in der Mitani-Sprache).

", , an Amenophis IV. Nr. 27-29.

", an Teie, Witwe Amenophis' III. Nr. 26.

Widia von Asḳalon an den König. 7 Briefe: Nr. 320-326.

Wiktazu an den König. Nr. 221. 222.

Zatatna von Akko an den König. Nr. 233. 234.

$Z\left[i_{-}{ }_{-}\right]$an den König. Nr. $33^{8}$.

Zî $[k] a r$, ein nordsyr. „Königssohn“, an den König von Ägypten. Nr. 44 .

$[Z]$ imrid $[a]$ an einen hohen (ägypt.) Beamten. Nr. 145.

Zimriddi von Șidon an den König. Mr. I44.

Zimridi von Lakiš an den König. Nr. 329.

Zišami $[m i]$ an Amenophis IV?. Nr. 210.

Zišamimi an den König. Nr. 209.

Zitatna an den König. Nr. 235.

Zitrijara an den König. Nr. 21 I-213.

Zurašar von $A[l]$ tirumna an den König. Nr. 319 .

Zurata von Akko an den König. Nr. 232.

_ - dih von Zuhra[ _?] an den König. Nr. 334.

[_ - von $Z] u k r[u]$ an den König. Nr. 335.

[- - h] epa, eine Frau, die wohl aus dem nördlichen bezw. nordöstlichen Syrien stammt (vgl. S. 20 unten), an ihre Herrin. Nr. 48.

_ _ - an den König. Nr. 339.

\section{Verzeichnisse von Geschenken:}

von Amenophis IV (kaum III) an [Burra]burariaš. Nr. 14.

aus Babylon, wohl von Burraburiaš. Nr. 13.

von Tušratta an Amenophis III. Nr. 22.

von Tušratta an Amenophis IV(?). Nr. 25.

Nr. 172? Vgl. oben unter r: Cölesyrische Briefe.

\section{Sonstige Tafeln:}

Mythologischen Inhalts. a) Adapa und der Südwind. Nr. 356;

b) Ereškigal und Nêrigal. Nr. 357 .

Unsicheren Inhalts. Nr. 340. 341. 358 .

Schreibübungen (vgl. S. 24). Nr. 345, vermutlich auch Nr. 346; ferner etwa auch Nr. 342-344. 347 .

Zeichenlisten verschiedener Art (auch diese sind zum Teil wohl Schülerarbeiten; vgl. S. 24). Nr. $348-354$.

\section{Tonzylinder}

mit dem Namen (Šamašniki) eines Tafelschreibers. Nr. 355. 